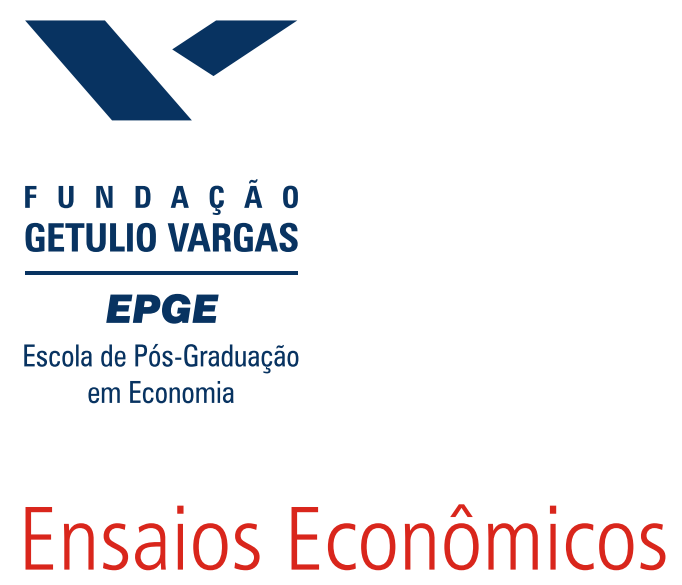

$$
\begin{aligned}
& \text { Escola de } \\
& \text { Pós Graduação } \\
& \text { em Economia } \\
& \text { da Fundação } \\
& \text { Getulio Vargas }
\end{aligned}
$$

On a Uniform Turnpike of the Third Kind in the Robinson-Solow-Srinivasan Model

M. Ali Khan, Alexander J. Zaslavski 
Os artigos publicados são de inteira responsabilidade de seus autores. As opiniões neles emitidas não exprimem, necessariamente, o ponto de vista da Fundação Getulio Vargas. 


\title{
ON A UNIFORM TURNPIKE OF THE THIRD KIND IN THE ROBINSON-SOLOW-SRINIVASAN MODEL
}

\author{
M. Ali Khan and Alexander J. Zaslavski
}

\begin{abstract}
On using McKenzie's taxonomy of optimal accumulation in the longrun, we report a "uniform turnpike" theorem of the third kind in a model original to Robinson, Solow and Srinivasan (RSS), and further studied by Stiglitz. Our results are presented in the undiscounted, discrete-time setting emphasized in the recent work of Khan-Mitra, and they rely on the importance of strictly concave felicity functions, or alternatively, on the value of a "marginal rate of transformation", $\xi_{\sigma}$, from one period to the next not being unity. Our results, despite their specificity, contribute to the methodology of intertemporal optimization theory, as developed in economics by Ramsey, von Neumann and their followers.
\end{abstract}

\section{INTRODUCTION}

In three lectures ${ }^{1}$ separated by a little over a quarter of a century, Lionel McKenzie $(1976,1998,1999)$ lifted the turnpike theorem from the narrower confines of the von Neumann model (1937), as originally conceived by Dorfman-Samuelson-Solow (1958), to a central position in the general theory of resource allocation over time. ${ }^{2}$ A crucial element in this move is the distinction between three kinds of turnpikes, with the third kind referring to a situation when growth paths are bunched together at the unforeseeable end, rather than at the beginning or "in the middle" of finite horizon programs. ${ }^{3}$ In his discussion of the "practical utility" of the three kinds of turnpike theorems, McKenzie invoked considerations pertaining to computability and limited information, and specifically in the context of the "late turnpike", he wrote:

1991 Mathematics Subject Classification. Journal of Economic Literature Classification Numbers: D90, C62, O21.

Key words and phrases. weakly maximal program, uniform turnpike, golden-rule program.

${ }^{1}$ The first two were delivered as the (i) Fisher-Schultz Lecture on September 1974 to The Econometric Society, (ii) the Richard T. Ely Lecture on May 1998 to The American Economic Association. The third has a somewhat more contorted history: initially composed at the request of the Journal of Economic Perspectives, it was delivered in December 1995 at Keio University as the acceptance lecture for the Rising Sun Medal, and a first version published in the Nagasaki Perfectural University Review (Vol 28, No. 1).

${ }^{2}$ The narrative is already encapsulated in the evolution of the three titles: from turnpike theory to turnpikes to the more general equilibrium, trade and capital accumulation. The full extent of the consolidation is evident in McKenzie (2002).

${ }^{3}$ Referring to the original Dorfman-Samuelson-Solow result, McKenzie (1976, p. 843) writes "The real ground for the result is the tendency for finite optimal paths to bunch together in the middle time, and this tendency is preserved even in models which are time-dependent." 
$[T]$ he convergence to one another of the infinite optimal paths from different initial stocks means that infinite optimal paths may be approximated by computing finite optimal paths with the stock of any (within limits) optimal path in some period $T$ as the target. This is useful if the infinite optimal path from a particular initial stock is easy to compute.

The basic point is that in situations when infinite programs can be approximated by finite ones of period $T$, the "significance of facts beyond period $T$ is fully allowed for in the choice of capital stocks for that period, ... and knowledge of tastes and technology beyond $T$ is not needed."

Thus, it bears renewed emphasis that even though McKenzie's 1976 taxonomy, and its tripartite temporal terminology in the form of early, middle and late turnpikes, has not been as universally subscribed to as it perhaps ought, ${ }^{4}$ the fundamental viewpoint it articulates is common to both the old and the newer theories of growth: planning in the immediate future by the market or an idealized, omniscient impartial observer, for capital accumulation in the long-run. ${ }^{5}$ And it is a point of view that is not limited to the original formulations of (middle) turnpikes of the first kind in a stationary multi-sectoral model without the possibility of optimizing consumption. Thus Brock's 1971 sensitivity analysis, as well as Dixit's 1970 comparison of the Fei-Ranis and Jorgenson's models of labor-surplus economies, can both be seen as a concern with circumstances pertaining the turnpike properties of the second kind, and modern investigations pertaining to asymptotic stability as a concern with turnpike properties of the third kind. The point is a bunching of trajectories, optimal or otherwise, early on, in the far future, or in their predominant middle, and stationarity of the model is hardly the primary consideration. ${ }^{6}$ However, it is the turnpike of the third kind that has received attention in recent work. Thus, even on limiting ourselves to the focus of this essay, strongly optimal programs without discounting, the importance of turnpike theory has been underscored for situations in which tastes and technology are not stationary or where the discount rate is variable, though under the terminological rubric of asymptotic stability rather than of turnpike theory. ${ }^{7}$

In this essay, in the context of a multi-sectoral model that dates to the sixties, we focus on strongly optimal programs in an undiscounted setting, and sharpen the question regarding the turnpike property of the third kind. In particular, we ask whether the golden-rule stock of such a model constitutes a uniform turnpike for

\footnotetext{
${ }^{4}$ McKenzie himself, in his 1998 article, refers to the turnpike of the first kind, the middle turnpike, as the Samuelson turnpike, and that of the third kind, the final turnpike, as the Ramsey turnpike.

${ }^{5}$ McKenzie (1998) looks at the "literature on optimal capital accumulation [and] the theory of competitive equilibrium over time" from the conceptual viewpoint of turnpike theory, and in particular, Sections VIII and IX of his lecture discuss endogenous growth theory subsequently surveyed in Aghion-Durlauf (2005).

${ }^{6}$ Thus McKenzie (1998; Section V) writes "[T]he fundamental convergence results ... do not depend on the presence of an optimal stationary path or the absence of changes in technology and taste."

${ }^{7}$ This, in the context of an aggregative model, Mitra (1979, p. 134) asks "When weakly maximal (or optimal) programs do exist, what can we say regarding their long-run behavior? [I]s there some sense in which such programs are asymptotically stable?" And Mitra-Zilcha (1981, p. 222) write "The asymptotic stability properties of weakly maximal and optimal programs are important as they show that optimal actions in the long-run are are invariant with respect to initial conditions of the economy (often called the "turnpike property")."
} 
its strongly optimal programs in the specific sense that given any arbitrarily small degree of approximation $\epsilon$, and given any two infinite optimal paths, does there exist a time period $T$ independent of the initial stocks of these programs such that they are $\epsilon$-close to each other beyond $T$ ? This question is important for precisely the reasons captured in the quote from McKenzie; given its importance, it asks whether the value of $T$ corresponding to an acceptable level of approximation remains the same irrespective of where the economy finds itself in a particular present. ${ }^{8}$ This is a question that has not been posed before in the context of the model that we study here, or, to our knowledge, even for strongly optimal programs in simple aggregative one- or two-good optimal growth models. ${ }^{9}$ We answer it in the affirmative in the form of Theorems 3.2 and 3.3 presented below.

The model that we work with here is due to Robinson (1960), Solow (1962) and Srinivasan (1962). It was originally motivated by the question of the "choice of technique' in so-called LDCs, and with the recent resurgence of interest in vintage capital, it has obvious relevance to the subject of innovation and embodied technical progress. But perhaps more to the point, even a rather special case of the model obtained with only a single type of machine, exhibits a veritable spectrum of dynamic phenomena, including cyclical and chaotic behavior. Thus the model is simple enough to be analytically tractable and complicated enough to shed light on canonical results in the theory of optimal accumulation, a potential complement perhaps to the other benchmark example due to Weitzman and reported in Samuelson (1973). Thus, the precise mathematical formulation of the question in the context of the rather particular RSS model, and its clean resolution, should not mislead the reader into thinking that this is a purely technical exercise of limited broader relevance, a filling of a theoretical lacuna solely for its own sake.

Leaving aside the possible practical importance of the concept of uniform turnpike, and its resolution for those of the first and second kinds, the RSS model is bound to prove to be an important benchmark in the general theory of intertemporal resource allocation.

It is clear however that the interest in and of a question does not necessarily translate into a difficulty and intricacy of its proof. We conclude the substantive part of this introduction by emphasizing that this is not the case here. Our proofs rely crucially on the structure of the arguments in Khan-Mitra (2005a) and in Zaslavski (2005), collected for the reader's convenience in Section 2. However, one needs substantial technical argument that goes beyond them; the auxiliary results presented in Section 5 develop these arguments by using the characterization of the von Neumann facet in Khan-Mitra (2005a) and the technique of making "time run backwards" exploited in Zaslavsky (2005). We leave it to the interested reader to understand for herself how much additional work needs to be done for the proofs of Theorem 3.2 even with the availability of these auxiliary results. The proof of Theorem 3.3 is a consequence of Theorem 3.2, and both of these proofs are presented in Section 6. Section 3 presents the statement of the principal three theorems of the paper, and Section 4 the proof of Theorem 3.1, a simpler consequence, relatively speaking, of results in Khan-Mitra and Zaslavski mentioned above.

for .

\footnotetext{
${ }^{8}$ In terms of the Mitra-Zilcha questions o footnote 7 above, we ask how invariant must the invariance to initial stocks be?

${ }^{9}$ There are of course results of this type in the mathematical literature of the calculus of variations and optimal control.
} 
(henceforth the RSS model) and further studied by Stiglitz $(1968,1973)$.

Khan-Mitra (2002, 2003 2005a, 2005b, 2005c) report results on the turnpike properties of discrete-time undiscounted optimal programs in the RSS model. In Mckenzie's terminology, they prove that the golden-rule stock (precise definitions to follow) is a turnpike of the third kind. Zaslavski (2005) extends some of the results of Khan-Mitra (2005a) in several directions, perhaps the most important being his focussed consideration of strongly optimal programs. ${ }^{10}$ His work makes it clear that Brock's (1970) example, and his pessimism regarding strongly optimal programs notwithstanding, this is a viable category for the RSS model, but under a parametric regime identified by Khan-Mitra in the discrete-time setting. In particular, Zaslavsky (2005) establishes the existence and turnpike properties of strongly optimal programs either when the felicity function is strictly concave, ${ }^{11}$ or when $\xi_{\sigma} \neq-1$, the parameter $\xi_{\sigma}$ being precisely the one identified in Khan-Mitra (2005a) and studied in some detail for the two-sector setting in Khan-Mitra (2005b and 2005c).

\section{The Model and Preliminary Results}

We begin with some preliminary notation. Let $R\left(R_{+}\right)$be the set of real (nonnegative) numbers. We shall work in a finite-dimensional Euclidean space $R^{n}$ with non-negative orthant $R_{+}^{n}=\left\{x \in R^{n}: x_{i} \geq 0, i=1, \ldots, n\right\}$. For any $x, y \in R^{n}$, let the inner product $x y=\sum_{i=1}^{n} x_{i} y_{i}$, and $x>>y, x>y, x \geq y$ have their usual meaning. Let $e(i), i=1, \ldots, n$, be the $i$ th unit vector in $R^{n}$, and $e$ be an element of $R_{+}^{n}$ all of whose coordinates are unity. For any $x \in R^{n}$, let $\|x\|$ denote the Euclidean norm of $x$.

We consider an economy capable of producing a finite number $n$ of alternative types of machines. For every $i=1, \ldots, n$, one unit of machine of type $i$ requires $a_{i}>0$ units of labor to construct it, and together with one unit of labor, each unit of it can produce $b_{i}>0$ units of a single consumption good. Thus, the production possibilities of the economy are represented by a labor input-coefficients vector, $a=\left(a_{1}, \ldots, a_{n}\right)>>0$ and an output-coefficients vector, $b=\left(b_{1}, \ldots, b_{n}\right)>>0$. Without loss of generality we assume that the types of machines are numbered such that $b_{1} \geq b_{2} \cdots \geq b_{n}$.

We shall assume that all machines depreciate at a rate $d \in(0,1)$. Thus the effective labor cost of producing a unit of output on a machine of type $i$ is given by $\left(1+d a_{i}\right) / b_{i}$ : the direct labor cost of producing unit output, and the indirect cost of replacing the depreciation of the machine in this production. We shall work with the reciprocal of the effective labor cost, the effective output that takes the depreciation into account, and denote it by $c_{i}$ for the machine of type $i$. Throughout this paper, we shall assume that there is a unique machine type $\sigma$ at which effective labor cost $\left(1+d a_{i}\right) / b_{i}$ is minimal, or at which the effective output per man $b_{i} /\left(1+d a_{i}\right)$ is maximal. Thus we shall assume:

(2.1) There exists $\sigma \in\{1, \ldots, n\}$ such that for all $i \in\{1, \ldots, n\} \backslash\{\sigma\}, c_{\sigma}>c_{i}$.

\footnotetext{
${ }^{10}$ There is an important issue of terminology here; Zaslavsky (2005) refers to the optimal programs in the work of Khan-Mitra as maximal programs, and to strongly optimal programs as simply optimal programs. In this, he follows McKenzie (2002, p. 256.) It is Zaslavsky's (and McKenzie's) terminology that we shall be following in our formal presentation, though not in this introduction.

${ }^{11}$ In this connection, there is also the earlier independent work, but in a two-sector setting, of Khan-Mitra (2002).
} 
For each nonnegative integer $t$ let $x(t)=\left(x_{1}(t), \ldots, x_{n}(t)\right) \geq 0$ denote the amounts of the $n$ types of machines that are available in time-period $t$, and let $z(t+1)=\left(z_{1}(t+1), \ldots, z_{n}(t+1)\right) \geq 0$ be the gross investments in the $n$ types of machines during period $(t+1)$. Hence, $z(t+1)=(x(t+1)-x(t))+d x(t)$, the sum of net investment and of depreciation. Let $y(t)=\left(y_{1}(t), \ldots, y_{n}(t)\right)$ be the amounts of the $n$ types of machines used for production of the consumption good, by $(t)$, during period $(t+1)$. Let the total labor force of the economy be stationary and positive. We shall normalize it to be unity. Clearly, gross investment, $z(t+1)$ representing the production of new machines of the various types, will require $a z(t+1)$ units of labor in period $t$. Also $y(t)$ representing the use of available machines for manufacture of the consumption good, will require $e y(t)$ units of labor in period $t$. Thus, the availability of labor constrains employment in the consumption and investment sectors by $a z(t+1)+e y(t) \leq 1$. Note that the flow of consumption and of investment (new machines) are in gestation during the period and available at the end of it. We now give a formal description of this technological structure.

Definition 2.1. A sequence $\{x(t), y(t)\}_{t=0}^{\infty}$ is called a program if for each integer $t \geq 0$

$$
\begin{gathered}
(x(t), y(t)) \in R_{+}^{n} \times R_{+}^{n}, x(t+1) \geq(1-d) x(t), \\
0 \leq y(t) \leq x(t), a(x(t+1)-(1-d) x(t))+e y(t) \leq 1 .
\end{gathered}
$$

Definition 2.2. Let $T_{1}, T_{2}$ be integers such that $0 \leq T_{1}<T_{2}$. A pair of sequences

$$
\left(\{x(t)\}_{t=T_{1}}^{T_{2}},\{y(t)\}_{t=T_{1}}^{T_{2}-1}\right)
$$

is called a program if $x\left(T_{2}\right) \in R_{+}^{n}$ and for each integer $t$ satisfying $T_{1} \leq t<T_{2}$ relations (2.2) hold.

Let $w:[0, \infty) \rightarrow R$ be a continuous strictly increasing concave and differentiable function which represents the preferences of the planner. Set

$$
\Omega=\left\{\left(x, x^{\prime}\right) \in R_{+}^{n} \times R_{+}^{n}: x^{\prime}-(1-d) x \geq 0 \text { and } a\left(x^{\prime}-(1-d) x\right) \leq 1\right\} .
$$

We have a correspondence $\Lambda: \Omega \rightarrow R_{+}^{n}$ given by

$$
\Lambda\left(x, x^{\prime}\right)=\left\{y \in R_{+}^{n}: 0 \leq y \leq x \text { and } e y \leq 1-a\left(x^{\prime}-(1-d) x\right)\right\} .
$$

For any $\left(x, x^{\prime}\right) \in \Omega$ define

$$
u\left(x, x^{\prime}\right)=\max \left\{w(b y\}: y \in \Lambda\left(x, x^{\prime}\right)\right\} .
$$

Definition 2.3. A golden-rule stock is $\widehat{x} \in R_{+}^{n}$ such that $(\widehat{x}, \widehat{x})$ is a solution to the problem: maximize $u\left(x, x^{\prime}\right)$ subject to (i) $x^{\prime} \geq x$; (ii)) $\left(x, x^{\prime}\right) \in \Omega$.

For the reader's convenience, we now reproduce some results from Khan-Mitra (2005a) and Zaslavski (2005).

Theorem 2.1. There exists a unique golden-rule stock $\widehat{x}=\left(1 /\left(1+d a_{\sigma}\right)\right) e(\sigma)$.

Proof: See the proof of Theorem 1 in Khan-Mitra (2005a).

It is not difficult to see that $\widehat{x}$ is a solution to the problem $w(b y) \rightarrow \max , y \in$ $\Lambda(\widehat{x}, \widehat{x})$. Set $\widehat{y}=\widehat{x}$, and for $i=1, \ldots, n$ set

$$
\widehat{q}_{i}=a_{i} b_{i} /\left(1+d a_{i}\right), \widehat{p}_{i}=w^{\prime}(b \widehat{x}) \widehat{q}_{i} .
$$

The following auxiliary result continues to have importance for the analysis of the RSS model. 
Lemma 2.1. $w(b \widehat{x}) \geq w(b y)+\widehat{p} x^{\prime}-\widehat{p} x$ for any $\left(x, x^{\prime}\right) \in \Omega$ and for any $y \in \Lambda\left(x, x^{\prime}\right)$.

Proof:. See the proof of Lemma 1 in Khan-Mitra (2005a).

Set the value-loss associated with any $\left(x, x^{\prime}\right) \in \Omega$ and any $y \in \Lambda\left(x, x^{\prime}\right)$ to be

$$
\delta\left(x, y, x^{\prime}\right)=\widehat{p}\left(x-x^{\prime}\right)-(w(b y)-w(b \widehat{y})) .
$$

By Lemma 2.1, $\delta\left(x, y, x^{\prime}\right) \geq 0$ for any $\left(x, x^{\prime}\right) \in \Omega$ and any $y \in \Lambda\left(x, x^{\prime}\right)$.

We use the following notion of good programs introduced by Gale (1967).

Definition 2.4. A program $\{x(t), y(t)\}_{t=0}^{\infty}$ is called good if there exists $M \in R$ such that for all $T \geq 0, \sum_{t=0}^{T}(w(b y(t))-w(b \widehat{y})) \geq M$. A program is called bad if $\lim _{T \rightarrow \infty} \sum_{t=0}^{T}(w(b y(t))-w(b \widehat{y}))=-\infty$.

The following general result of Gale's applies to the RSS model.

Proposition 2.1. Any program that is not good is bad.

Proof: See the proof of Proposition 4 in Khan-Mitra (2005a).

The fact that there exist good programs in the RSS model for any initial stock is established in Proposition 2 in Khan-Mitra (2005a). They also established

Proposition 2.2. A program $\{x(t), y(t)\}_{t=0}^{\infty}$ is good if and only if

$$
\sum_{t=0}^{\infty} \delta(x(t), y(t), x(t+1))<\infty
$$

Proof: See the proof of Proposition 7 in Khan-Mitra (2005a).

Let $x_{0} \in R_{+}^{n}$. Set

$$
\Delta\left(x_{0}\right)=\inf \left\{\sum_{t=0}^{\infty} \delta(x(t), y(t), x(t+1)):\{x(t), y(t)\}_{t=0}^{\infty} \text { a program from } x_{0}\right\}
$$

Since there exists a good program from $x_{0}$ it follows from Proposition 2.2 that $\Delta\left(x_{0}\right)<\infty$. The following result can now be established.

Proposition 2.3. Let $x_{0} \in R_{+}^{n}$. Then there exists a program $\{x(t), y(t)\}_{t=0}^{\infty}$ from $x_{0}$ such that

$$
\sum_{t=0}^{\infty} \delta(x(t), y(t), x(t+1))=\Delta\left(x_{0}\right) .
$$

Proof: See the proof of Proposition 8 in Khan-Mitra (2005a).

Let

$$
\xi_{\sigma}=1-d-\left(1 / a_{\sigma}\right) .
$$

A distinguishing characteristic of the RSS model is that good programs satisfy the turnpike property under two distinct regimes: (i) strictly concave felicity functions (ii) $\xi_{\sigma} \neq-1$. This was established in the two-sector version of the RSS model in Khan-Mitra (2002, 2005b), and in the general case (with an alternative, and powerful argument) by Zaslavski (2005). The introduction, and geometric significance, of $\xi_{\sigma}$ is made explicit in Khan-Mitra (2005a). 
Theorem 2.2. Let the function $w$ be strictly concave. Then for each good program $\{x(t), y(t)\}_{t=0}^{\infty}$,

$$
\lim _{t \rightarrow \infty}(x(t), y(t))=(\widehat{x}, \widehat{x}) .
$$

Proof: See the proof of Theorem 2.2 in Zaslavski (2005).

Theorem 2.3. Let $\xi_{\sigma} \neq-1$. Then for each good program $\{x(t), y(t)\}_{t=0}^{\infty}$,

$$
\lim _{t \rightarrow \infty}(x(t), y(t))=(\widehat{x}, \widehat{x})
$$

Proof:. See the proof of Theorem 2.3 in Zaslavski (2005).

In the sequel we use a notion of an optimal (overtaking) program introduced in Atsumi (1965) and von-Weizsacker (1965) and first studied in a multi-sectoral context by Gale (1967). We also use the notion of a maximal program introduced in Brock (1970). ${ }^{12}$

Definition 2.5. A program $\left\{x^{*}(t), y^{*}(t)\right\}_{t=0}^{\infty}$ is called optimal if

$$
\limsup _{T \rightarrow \infty} \sum_{t=0}^{T}\left[w(b y(t))-w\left(b y^{*}(t)\right)\right] \leq 0
$$

for every program $\{x(t), y(t)\}_{t=0}^{\infty}$ satisfying $x(0)=x^{*}(0)$.

Definition 2.6. A program $\left\{x^{*}(t), y^{*}(t)\right\}_{t=0}^{\infty}$ is called maximal if for each program $\{x(t), y(t)\}_{t=0}^{\infty}$ satisfying $x(0)=x^{*}(0)$ the following relation holds:

$$
\liminf _{T \rightarrow \infty} \sum_{t=0}^{T}\left[w(b y(t))-w\left(b y^{*}(t)\right)\right] \leq 0 .
$$

Khan and Mitra (2005a, Theorem 2) established that there exists in the RSS model a maximal program starting from any initial stock. Zaslavski (2005) generalized their result to optimal programs.

Theorem 2.4. Assume that for each good program $\{x(t), y(t)\}_{t=0}^{\infty}$

$$
\lim _{t \rightarrow \infty}(x(t), y(t))=(\widehat{x}, \widehat{x}) .
$$

Then for each initial stock $x_{0} \in R_{+}^{n}$ there exists an optimal program $\{x(t), y(t)\}_{t=0}^{\infty}$ which satisfies $x(0)=x_{0}$.

Proof: See the proof of Corollaries 2.2 and 2.3 in Zaslavskyi (2005).

\section{MAIN RESUlts}

In this paper we will establish the following three results.

\footnotetext{
${ }^{12}$ Note that in the introduction maximal and optimal programs are being referred to as weakly maximal and strongly optimal programs respectively.
} 
Theorem 3.1. Assume that for each good program $\{u(t), v(t)\}_{t=0}^{\infty}$

$$
\lim _{t \rightarrow \infty}(u(t), v(t))=(\widehat{x}, \widehat{x}) .
$$

Then for each program $\{x(t), y(t)\}_{t=0}^{\infty}$ the following conditions are equivalent:

(i) $\sum_{t=0}^{\infty} \delta(x(t), y(t), x(t+1))=\Delta(x(0))$.

(ii) $\{x(t), y(t)\}_{t=0}^{\infty}$ is optimal.

(iii) $\{x(t), y(t)\}_{t=0}^{\infty}$ is maximal.

Theorem 3.2. Assume that at least one of the following conditions holds:

(a) $w$ is strictly concave.

(b) $\xi_{\sigma} \neq-1$.

Let $\epsilon>0$. Then there exists $\delta>0$ such that for each optimal program $\{x(t), y(t)\}_{t=0}^{\infty}$ satisfying $\|x(0)-\widehat{x}\| \leq \delta$ the following inequality holds:

$$
\|x(t)-\widehat{x}\|,\|y(t)-\widehat{x}\| \leq \epsilon
$$

for all integers $t \geq 0$.

Theorem 3.3. Assume that at least one of the following conditions holds:

(a) $w$ is strictly concave.

(b) $\xi_{\sigma} \neq-1$.

Let $M_{0}, \epsilon>0$. Then there exists a natural number $T_{0}$ such that for each optimal program $\{x(t), y(t)\}_{t=0}^{\infty}$ satisfying $x(0) \leq M_{0} e$ and each integer $t \geq T_{0}$

$$
\|x(t)-\widehat{x}\|,\|y(t)-\widehat{x}\| \leq \epsilon .
$$

\section{Proof of Theorem 3.1}

Proof. Let $\{x(t), y(t)\}_{t=0}^{\infty}$ be a program. the fact that (i) implies (ii) follows from the proof of Theorem 2.4 In Zaslavskyi (2005). Evidently (ii) implies (iii). Let us show that (iii) implies (i).

Assume that $\{x(t), y(t)\}_{t=0}^{\infty}$ is maximal. By Khan-Mitra (2004, Proposition 10) $\{x(t), y(t)\}_{t=0}^{\infty}$ is good. Proposition 2.2 implies that

$$
\sum_{t=0}^{\infty} \delta(x(t), y(t), x(t+1))<\infty
$$

In view of Proposition 2.3 there exists a program $\left\{x^{*}(t), y^{*}(t)\right\}_{t=0}^{\infty}$ such that

$$
x^{*}(0)=x(0), \sum_{t=0}^{\infty} \delta\left(x^{*}(t), y^{*}(t), x^{*}(t+1)\right)=\Delta(x(0)) .
$$

Since $\{x(t), y(t)\}_{t=0}^{\infty}$ and $\left\{x^{*}(t), y^{*}(t)\right\}_{t=0}^{\infty}$ are good programs we have

$$
\lim _{t \rightarrow \infty}(x(t), y(t))=\lim _{t \rightarrow \infty}\left(x^{*}(t), y^{*}(t)\right)=(\widehat{x}, \widehat{x}) .
$$

By Khan-Mitra (2004, Proposition 6) and (4.2) for all integers $T \geq 0$

$$
\sum_{t=0}^{T}(w(b y(t))-w(b \widehat{y}))=\widehat{p}(x(0)-x(T+1))-\sum_{t=0}^{\infty} \delta(x(t), y(t), x(t+1)),
$$




$$
\sum_{t=0}^{T}\left(w\left(b y^{*}(t)\right)-w(b \widehat{y})\right)=\widehat{p}\left(x^{*}(0)-x^{*}(T+1)\right)-\sum_{t=0}^{T} \delta\left(x^{*}(t), y^{*}(t), x^{*}(t+1)\right)
$$

and

$$
\begin{gathered}
\sum_{t=0}^{T}\left(w\left(b y^{*}(t)\right)-w(b y(t))=\widehat{p}\left(x(T+1)-x^{*}(T+1)\right)\right. \\
+\sum_{t=0}^{T} \delta(x(t), y(t), x(t+1))-\sum_{t=0}^{\infty} \delta\left(x^{*}(t), y^{*}(t), x^{*}(t+1)\right) .
\end{gathered}
$$

Since the program $\{x(t), y(t)\}_{t=0}^{\infty}$ is maximal it follows from (4.1)-(4.4) that

$$
\begin{gathered}
0 \geq \liminf _{T \rightarrow \infty} \sum_{t=0}^{T}\left[w\left(b y^{*}(t)\right)-w(b y(t))\right]= \\
=\sum_{t=0}^{\infty} \delta(x(t), y(t), x(t+1))-\sum_{t=0}^{\infty} \delta\left(x^{*}(t), y^{*}(t), x^{*}(t+1)\right) \\
+\widehat{p} \lim _{T \rightarrow \infty}\left(x(T+1)-x^{*}(T+1)\right)=\sum_{t=0}^{\infty} \delta(x(t), y(t), x(t+1))-\Delta x(0) .
\end{gathered}
$$

Thus from (2.4)

$$
\sum_{t=0}^{\infty} \delta(x(t), y(t), x(t+1))=\Delta x(0) .
$$

This completes the proof of the theorem.

\section{Auxiliary Results for Theorems 3.2 And 3.3}

We use two results from Khan-Mitra (2005a) and another from Zaslavski (2005) as the basis for the results presented in this section.

Proposition 5.1. Let $m_{0}>0$. Then there is $m_{1}>0$ such that for each natural number $T$ and each program $\left(\{x(t)\}_{t=0}^{T},\{y(t)\}_{t=0}^{T-1}\right)$ which satisfies $x(0) \leq m_{0} e$ the inequality $x(t) \leq m_{1}$ e holds for all integers $t \in[0, T]$.

Proof: See the proof of Proposition 1 in Khan-Mitra (2005a).

Proposition 5.2. The von Neumann facet

$$
\left\{\left(x, x^{\prime}\right) \in \Omega: \text { there is } y \in \Lambda\left(x, x^{\prime}\right) \text { such that } \delta\left(x, y, x^{\prime}\right)=0\right\}
$$

is a subset of

$$
\begin{gathered}
\left\{\left(x, x^{\prime}\right) \in \Omega: x_{i}^{\prime}=x_{i}=0 \text { for all } i \in\{1, \ldots, n\} \backslash\{\sigma\},\right. \\
\left.x_{\sigma}^{\prime}=\left(1 / a_{\sigma}\right)+\xi_{\sigma} x_{\sigma}\right\},
\end{gathered}
$$

with equality if the function $w$ is linear. If the function $w$ is strictly concave, then the facet is the singleton $\{(\widehat{x}, \widehat{x})\}$.

Proof: See the proof of Lemma 2 in Khan-Mitra (2005a). 
Proposition 5.3. Let $\xi_{\sigma} \neq-1$ and let a sequence $\{x(t), y(t)\}_{t=-\infty}^{\infty} \subset R_{+}^{2 n}$ satisfy the following conditions:

There is $S_{0}>0$ such that

$$
\begin{gathered}
\|x(t)\|,\|y(t)\| \leq S_{0} \text { for all integers } t \\
x(t+1) \geq(1-d) x(t) \text { and } 0 \leq y(t) \leq x(t) \text { for all integers } t \\
a(x(t+1)-(1-d) x(t))+e y(t) \leq 1 \text { for all integers } t \\
\delta(x(t), y(t), x(t+1))=0 \text { for all integers } t .
\end{gathered}
$$

Then

$$
x(t)=y(t)=\widehat{x} \text { for all integers } t .
$$

Proof:. See the proof of Lemma 4.1 in Zaslavski (2005).

Proposition 5.4. Let $M_{0}>0$. Then $\sup \left\{\Delta(x): x \in R_{+}^{n}\right.$ and $\left.x \leq M_{0} e\right\}<\infty$.

Proof. By Proposition 5.1 there is $M_{1}>0$ such that for each program $\{x(t), y(t)\}_{t=0}^{\infty}$ satisfying $x(0) \leq M_{0} e$ we have $x(t) \leq M_{1} e$ for each integers $t \geq 0$. Set

$$
z(0)=0, z(t+1)=(1-d) z(t)+\left(1 / a_{\sigma}\right) e(\sigma), t=0,1, \ldots
$$

It is easy to see that

$$
\lim _{T \rightarrow \infty} z(T)=\left[a_{\sigma}^{-1} \sum_{t=0}^{T-1}(1-d)^{t}\right] e(\sigma)=\left(d a_{\sigma}\right)^{-1} e(\sigma) .
$$

Clearly there exists a natural number $T_{0}$ such that

$$
z\left(T_{0}\right) \geq \widehat{x}
$$

Assume now that $x \in R_{+}^{n}$ satisfies $x \leq M_{0} e$. Set

$$
\begin{gathered}
x(0)=x, x(t+1)=(1-d) x(t)+\left(1 / a_{\sigma}\right) e(\sigma), t=0, \ldots, T_{0}-1, \\
y(t)=0, t=0, \ldots, T_{0}-1 .
\end{gathered}
$$

It is not difficult to see that $\left(\{x(t)\}_{t=0}^{T_{0}},\{y(t)\}_{t=0}^{T_{0}-1}\right)$ is a program and

$$
x(t) \geq z(t), t=0, \ldots, T_{0} .
$$

By (5.5) and (5.3)

$$
x\left(T_{0}\right) \geq z\left(T_{0}\right) \geq \widehat{x} .
$$

For all natural numbers $t \geq T_{0}$ set

$$
y(t)=\widehat{x}, x(t+1)=(1-d) x(t)+d \widehat{x} .
$$


It is easy to see that $\{x(t), y(t)\}_{t=0}^{\infty}$ is a program. In view of Khan-Mitra (2004, Proposition 6) and (5.7) for all natural numbers $T>T_{0}$,

$$
\begin{gathered}
\sum_{t=0}^{T} \delta(x(t), y(t), x(t+1))=\widehat{p}(x(0)-x(T+1))-\sum_{t=0}^{T}(w(b y(t))-w(b \widehat{y})) \\
\leq M_{0} \widehat{p} e-\sum_{t=0}^{T_{0}-1}[w(b y(t))-w(b \widehat{y})] .
\end{gathered}
$$

It follows from (5.8) that for all natural numbers $T>T_{0}$

$$
\sum_{t=0}^{T} \delta(x(t), y(t), x(t+1)) \leq M_{0} \widehat{p} e+T_{0} w(b \widehat{y}) .
$$

This inequality implies that

$$
\Delta(x) \leq \sum_{t=0}^{\infty} \delta(x(t), y(t), x(t+1)) \leq M_{0} \widehat{p} e+T_{0} w(b \widehat{y}) .
$$

This completes the proof of the proposition.

Proposition 5.5. Let $\epsilon>0$. Then there exists $\delta>0$ such that for each $x, x^{\prime} \in R_{+}^{n}$ satisfying

$$
\|x-\widehat{x}\|,\left\|x^{\prime}-\widehat{x}\right\| \leq \delta
$$

there exist $\bar{x} \geq x^{\prime}, y \in R_{+}^{n}$ such that

$$
\begin{gathered}
(x, \bar{x}) \in \Omega, y \in \Lambda(x, \bar{x}), \\
\|y-\widehat{x}\| \leq \epsilon,\|\bar{x}-\widehat{x}\| \leq \epsilon .
\end{gathered}
$$

Proof. Choose a positive number $\delta$ such that

$$
16 \delta n+2 \delta\|a\| n<\min \left\{\epsilon, 8^{-1} d\left(1+d a_{\sigma}\right)^{-1}\right\} .
$$

Let $x, x^{\prime} \in R_{+}^{n}$ satisfy

$$
\|x-\widehat{x}\|,\left\|x^{\prime}-\widehat{x}\right\| \leq \delta .
$$

Define $z \in R^{n}$ as follows:

$$
\begin{gathered}
z_{i}=\max \left\{x_{i}^{\prime}-(1-d) x_{i}, 0\right\}, i=1, \ldots n, \\
y=\min \left\{x_{\sigma},(1-a z)\right\} e(\sigma), \bar{x}=(1-d) x+z .
\end{gathered}
$$

By (5.12) and (5.11)

$$
\bar{x} \geq(1-d) x, \bar{x} \geq x^{\prime} .
$$


In view of (5.12)

$$
a(\bar{x}-(1-d) x)=a z .
$$

It follows from (5.10) and (5.11) that for each $i \in\{1, \ldots, n\} \backslash\{\sigma\}$

$$
\left|x_{i}^{\prime}\right|,\left|x_{i}\right| \leq \delta,-\delta \leq x_{i}^{\prime}-(1-d) x_{i} \leq \delta, 0 \leq z_{i} \leq \delta .
$$

By (5.10)

$$
\begin{gathered}
\left|\left[x_{\sigma}^{\prime}-(1-d) x_{\sigma}\right]-d\left(d a_{\sigma}+1\right)^{-1}\right| \\
\leq\left|x_{\sigma}^{\prime}-\left(1+d a_{\sigma}\right)^{-1}\right|+(1-d)\left|x_{\sigma}-\left(d a_{\sigma}+1\right)^{-1}\right| \leq 2 \delta .
\end{gathered}
$$

Together with (5.9) and (5.11) this inequality implies that

$$
\begin{gathered}
x_{\sigma}^{\prime}-(1-d) x_{\sigma}>0, z_{\sigma}=x_{\sigma}^{\prime}-(1-d) x_{\sigma}, \\
\left|z_{\sigma}-d\left(1+d a_{\sigma}\right)^{-1}\right| \leq 2 \delta .
\end{gathered}
$$

In view of (5.12), (5.10), (5.15) and (5.9)

$$
\|\bar{x}-\widehat{x}\| \leq\|(1-d) x-(1-d) \widehat{x}\|+\|z-d \widehat{x}\| \leq
$$

$$
\delta+\|z-d \widehat{x}\| \leq \delta+2 \delta n<\epsilon
$$

It follows from (5.16), (5.15) and (5.9) that

$$
|a z-a d \widehat{x}| \leq\|a|||| z-d \widehat{x}|| \leq\| a|| 2 \delta n<8^{-1}\left(1+d a_{\sigma}\right)^{-1}
$$

and

$$
a z \leq a d \widehat{x}+8^{-1}\left(1+d a_{\sigma}\right)^{-1}=a_{\sigma} d\left(1+d a_{\sigma}\right)^{-1}=\left(8\left(1+d a_{\sigma}\right)\right)^{-1}<1 .
$$

Combined with (5.14) and (5.13) this relation implies that

$$
a(\bar{x}-(1-d) x)<1 \text { and }(x, \bar{x}) \in \Omega .
$$

By (5.12), (5.20), (5.19) and (5.14) $y \in \Lambda(x, \bar{x})$. In view of (5.12), (5.10), (5.18) and (5.9)

$$
\begin{gathered}
|| y-\widehat{x}||=|| y_{\sigma}-\widehat{x}_{\sigma}|| \leq\left|1-a z-\left(1+d a_{\sigma}\right)^{-1}\right|+\left|x_{\sigma}-\widehat{x}_{\sigma}\right| \\
\leq \delta+\left|d a_{\sigma}\left(1+d a_{\sigma}\right)^{-1}-a z\right|=\delta+|a z-a d \widehat{x}| \leq \delta+|| a|| 2 \delta n<\epsilon .
\end{gathered}
$$

This completes the proof of the proposition. 
Proposition 5.6. Assume that for each good program $\{x(t), y(t)\}_{t=0}^{\infty}$,

$$
\lim _{t \rightarrow \infty}(x(t), y(t))=(\widehat{x}, \widehat{x}) .
$$

Let $\epsilon>0$. Then there is $\delta>0$ such that for each $x \in R_{+}^{n}$ satisfying $\|x-\widehat{x}\| \leq \delta$ the inequality $\Delta(x) \leq \epsilon$ holds.

Proof. Choose a positive number $\delta_{1}$ such that

$$
\begin{gathered}
\delta_{1}\|\widehat{p}\|<\epsilon / 8 \text { and } \delta_{1}<\epsilon / 8, \\
|w(b y)-w(b \widehat{y})| \leq \epsilon / 8 \text { for each } y \in R_{+}^{n} \text { satisfying }\|y-\widehat{y}\| \leq \delta_{1} .
\end{gathered}
$$

By Proposition 5.5 there is $\delta \in\left(0, \delta_{1} / 4\right)$ such that for each $x, x^{\prime} \in R_{+}^{n}$ satisfying $\|x-\widehat{x}\|,\left\|x^{\prime}-\widehat{x}\right\| \leq \delta$ there exist $\bar{x} \geq x^{\prime}, y \in R_{+}^{n}$ such that

$$
(x, \bar{x}) \in \Omega, y \in \Lambda(x, \bar{x}),\|y-\widehat{x}\|,\|\bar{x}-\widehat{x}\| \leq \delta_{1} / 4 .
$$

Let $x \in R_{+}^{n}$ satisfy

$$
\|x-\widehat{x}\| \leq \delta
$$

In view of this inequality and the choice of $\delta$ there are $\bar{x} \in R_{+}^{n}, y \in R_{+}^{n}$ such that

$$
\begin{gathered}
(x, \bar{x}) \in \Omega, y \in \Lambda(x, \bar{x}), \\
\bar{x} \geq \widehat{x},\|\bar{x}-\widehat{x}\|,\|y-\widehat{y}\| \leq \delta_{1} / 4 .
\end{gathered}
$$

Define

$$
\begin{gathered}
x(0)=x, y(0)=y, x(1)=\bar{x}, \\
x(t+1)=(1-d) x(t)+d \widehat{x}, y(t)=\widehat{y}
\end{gathered}
$$

for all integers $t \geq 1$. Relations (5.25) and (5.26) imply that $\{x(t), y(t)\}_{t=0}^{\infty}$ is a good program and

$$
\lim _{t \rightarrow \infty} x(t)=\widehat{x} .
$$

By Khan-Mitra (2004, Proposition 6), (5.24)-(5.27), (5.21) and (5.22)

$$
\begin{gathered}
\Delta(x) \leq \sum_{t=0}^{\infty} \delta(x(t), y(t), x(t+1))=\lim _{T \rightarrow \infty}\left[\widehat{p}(x(0)-x(T+1))-\sum_{t=0}^{T}(w(b y(t))-w(b \widehat{y}))\right] \\
=\widehat{p}(x-\widehat{x})-(w(b y(0))-w(b \widehat{y})) \leq|| \widehat{p}|| \delta+|w(b y(0))-w(b \widehat{y})| \\
\leq\|\widehat{p}\| \delta+|w(b y)-w(b \widehat{y})|<\epsilon / 2 .
\end{gathered}
$$

The proposition is proved. 
6. Proofs of Theorems 3.2 and 3.3

Lemma 6.1. Let $S_{0}, \epsilon>0$. Then there exists $\delta>0$ such that for each sequence $\{x(t), y(t)\}_{t=0}^{\infty} \subset R_{+}^{2 n}$ satisfying

$$
\|x(t)\|,\|y(t)\| \leq S_{0} \text { for all integers } t
$$

$$
\begin{gathered}
(x(t), x(t+1)) \in \Omega, y(t) \in \Lambda(x(t), x(t+1)) \text { for all integers } t, \\
\delta(x(t), y(t), x(t+1)) \leq \delta \text { for all integers } t
\end{gathered}
$$

the following inequality holds:

$$
\|x(t)-\widehat{x}\|,\|y(t)-\widehat{x}\| \leq \epsilon \text { for all integers } t .
$$

Proof. Let us assume the converse. Then for each natural number $k$ there exist a sequence $\left\{x^{(k)}(t), y^{(k)}(t)\right\}_{t=-\infty}^{\infty} \subset R_{+}^{2 n}$ which satisfies

$$
\begin{gathered}
\left\|x^{(k)}(t)\right\|,\left\|y^{(k)}(t)\right\| \leq S_{0} \text { for all integers } t, \\
\left(x^{(k)}(t), x^{(k)}(t+1)\right) \in \Omega \text { for all integers } t, \\
\delta\left(x^{(k}(t), y^{(k)}(t), x^{(k)}(t+1)\right) \leq 1 / k \text { for all integers } t
\end{gathered}
$$

and an integer $\tau_{k}$ such that

$$
\max \left\{\left\|x^{(k)}\left(\tau_{k}\right)-\widehat{x}\right\|,\left\|y^{(k)}\left(\tau_{k}\right)-\widehat{x}\right\|\right\} \geq \epsilon .
$$

We may assume without loss of generality that

$$
\tau_{k}=0 \text { for all natural numbers } k \text {. }
$$

Extracting subsequences, re-indexing and using diagonalization process we obtain that there exists a strictly increasing sequence of natural numbers $\left\{k_{j}\right\}_{j=1}^{\infty}$ such that for each integer $s$ there is

$$
(u(s), v(s))=\lim _{j \rightarrow \infty}\left(x^{\left(k_{j}\right)}(s), y^{\left(k_{j}\right)}(s)\right) .
$$

Since the set

$$
\left\{\left(x, y, x^{\prime}\right) \in R_{+}^{3 n}:\left(x, x^{\prime}\right) \in \Omega, y \in \Lambda\left(x, x^{\prime}\right)\right\}
$$

is closed it follows from (6.10) and (6.6) that

$$
(u(s), u(s+1)) \in \Omega, v(s) \in \Lambda(u(s), u(s+1)) \text { for all integers } s .
$$


In view of (6.5) and (6.10) for all integers $s$

$$
\|u(s)\|,\|v(s)\| \leq S_{0} .
$$

Since the function $\delta(\cdot, \cdot, \cdot)$ is nonnegative and continuous it follows from (6.10) and (6.7) that

$$
\delta(u(s), v(s), u(s+1))=0 \text { for all integers } s .
$$

Relations (6.8)-(6.10) imply that

$$
\max \{\|u(0)-\widehat{x}\|,\|v(0)-\widehat{x}\|\} \geq \epsilon .
$$

If $w$ is strictly concave, then it follows from $(6.11),(6.13)$ and Proposition 5.2 that

$$
u(s)=v(s)=\widehat{x} \text { for all integers } s .
$$

If $\xi_{\sigma} \neq-1$, then it follows from (6.11)-(6.13) and Proposition 5.3 that for all integers $s, u(s)=v(s)=\widehat{x}$. Thus (6.15) holds in both cases. This contradicts (6.14). The contradiction we have reached proves the lemma.

Choose

$$
M_{0}>1+\left(1+d a_{\sigma}\right)^{-1} .
$$

Proof of Theorem 3.2. By Proposition 5.1 there is $S_{0}>0$ such that for each program $\{x(t), y(t)\}_{t=0}^{\infty}$ satisfying $x(0) \leq M_{0} e$,

$$
x(t) \leq S_{0} e \text { for all integers } t \geq 0 .
$$

In view of Lemma 6.1 there is $\epsilon_{1} \in(0, \epsilon)$ such that for each sequence $\{x(t), y(t)\}_{t=-\infty}^{\infty} \subset$ $R_{+}^{2 n}$ satisfying

$$
\|x(t)\|,\|y(t)\| \leq S_{0} n \text { for all integers } t
$$

$$
(x(t), x(t+1)) \in \Omega, y(t) \in \Lambda(x(t), x(t+1)), \delta(x(t), y(t), x(t+1)) \leq \epsilon_{1}
$$

for all integers $t$, the following inequality holds:

$$
\|x(t)-\widehat{x}\|,\|y(t)-\widehat{y}\| \leq \epsilon / 8 \text { for all integers } t \text {. }
$$

By Proposition 5.6 and Theorems 2.2 and 2.3 there is $\delta_{1}>0$ such that for each $x \in R_{+}^{n}$ satisfying $\|x-\widehat{x}\| \leq \delta_{1}$,

$$
\Delta(x) \leq \epsilon_{1} / 4 .
$$

It follows from Proposition 5.5 and the continuity of the function $\delta(\cdot, \cdot, \cdot)$ that there is $\delta_{2}>0$ such that for each $x \in R_{+}^{n}$ satisfying $\|x-\widehat{x}\| \leq \delta_{2}$ there exist $\bar{x} \geq x$ and $y \in R_{+}^{n}$ which satisfy

$$
(\widehat{x}, \bar{x}) \in \Omega, y \in \Lambda(\widehat{x}, \bar{x})
$$




$$
\delta(\widehat{x}, y, \bar{x}) \leq \epsilon_{1} / 4,\|\bar{x}-x\| \leq\left(\epsilon_{1} / 4\right)(\|\widehat{p}\|+1)^{-1} .
$$

Set

$$
\delta=\min \left\{\delta_{1}, \delta_{2}, 1\right\}
$$

Assume that $\{x(t), y(t)\}_{t=0}^{\infty}$ is an optimal program such that

$$
\|x(0)-\widehat{x}\| \leq \delta
$$

By Theorems 2.2, 2.3 and 3.1, the choice of $\delta_{1}$ (see (6.21)), (6.24) and (6.23)

$$
\sum_{t=0}^{\infty} \delta(x(t), y(t), x(t+1))=\Delta(x(0)) \leq \epsilon_{1} / 4 .
$$

In view of $(6.24),(6.23)$ and the choice of $\delta_{2}$ (see (6.22)) there exist $\bar{x} \geq x(0)$ and $\bar{y} \in R_{+}^{n}$ such that

$$
\begin{gathered}
(\widehat{x}, \bar{x}) \in \Omega, \bar{y} \in \Lambda(\widehat{x}, \bar{x}), \delta(\widehat{x}, \bar{y}, \bar{x}) \leq \epsilon_{1} / 4 \\
\|\bar{x}(0)-x(0)\| \leq\left(\epsilon_{1} / 4\right)(\|\widehat{p}\|+1)^{-1}
\end{gathered}
$$

Set

$$
z=\bar{x}-x(0)
$$

Define a sequence $\{\bar{x}(t), \bar{y}(t)\}_{t=-\infty}^{\infty}$ by

$$
\begin{gathered}
\bar{x}(t)=\widehat{x} \text { for all integers } t<0, \\
\bar{y}(t)=\widehat{x} \text { for all integers } t<-1, \\
\bar{y}(-1)=\bar{y}, \bar{x}(0)=\bar{x}, \\
\bar{x}(t)=x(t)+(1-d)^{t} z \text { for all integers } t \geq 1, \\
\bar{y}(t)=y(t) \text { for all integers } t \geq 0 .
\end{gathered}
$$

It follows from (6.28), (6.26) and (6.27) that

$$
(\bar{x}(t), \bar{x}(t+1)) \in \Omega, \bar{y}(t) \in \Lambda(\bar{x}(t), \bar{x}(t+1)) \text { for all integers } t .
$$

By (6.29), (6.28), (6.16) and the choice of $S_{0}($ see $(6.17))$

$$
\|\bar{x}(t)\|,\|\bar{y}(t)\| \leq S_{0} n \text { for all integers } t .
$$

In view of (6.28), (6.25) and Theorems 2.2 and 2.3

$$
\lim _{i \rightarrow \infty} \bar{x}(t)=\lim _{t \rightarrow \infty} x(t)=\widehat{x} .
$$


Khan-Mitra (2004, Proposition 6) and (6.28) imply that for any natural number $T$

$$
\begin{gathered}
\sum_{t=0}^{T} \delta(\bar{x}(t), \bar{y}(t), \bar{x}(t+1))=\widehat{p}(\bar{x}(0)-\bar{x}(T+1))-\sum_{t=0}^{T}(w(b \bar{y}(t))-w(b \widehat{y})) \\
=\widehat{p}(x(0)-x(T+1))-\sum_{t=0}^{T}(w(b y(t))-w(b \widehat{y})) \\
+\widehat{p}(\bar{x}(0)-x(0))+\widehat{p}(x(T+1)-\bar{x}(T+1)) \\
\leq \sum_{t=0}^{T} \delta(x(t), y(t), x(t+1))+\|\widehat{p}\|\|\bar{x}(0)-x(0)\|+\|\widehat{p}\|\|x(T+1)-\bar{x}(T+1)\| .
\end{gathered}
$$

Combined with (6.31), (6.25) and (6.26) this relation implies that

$\sum_{t=0}^{\infty} \delta(\bar{x}(t), \bar{y}(t), \bar{x}(t+1)) \leq \sum_{t=0}^{\infty} \delta(x(t), y(t), x(t+1))+\|\widehat{p}\|\|\bar{x}(0)-x(0)\| \leq \epsilon_{1} / 4+\epsilon_{1} / 4$.

It follows from (6.32), (6.28) and (6.26) that for all integers $t$

$$
\delta(\bar{x}(t), \bar{y}(t), \bar{x}(t+1)) \leq \epsilon_{1} .
$$

In view of (6.33), (6.29), (6.30) and the choice of $\epsilon_{1}$ (see (6.18)-(6.20))

$$
\|\bar{x}(t)-\widehat{x}\|,\|\bar{y}(t)-\widehat{x}\| \leq \epsilon / 8
$$

for all integers $t$. Relations (6.26)-(6.28) imply that for all integers $t \geq 0$

$$
\|\bar{x}(t)-x(t)\| \leq \epsilon_{1} / 4<\epsilon / 4 .
$$

By (6.34) and (6.28) for all integers $t \geq 0$

$$
\|y(t)-\widehat{x}\| \leq \epsilon / 8 .
$$

In view of (6.34) and (6.35) for all integers $t \geq 0$

$$
\|x(t)-\widehat{x}\| \leq\|x(t)-\bar{x}(t)\|+\|\bar{x}(t)-\widehat{x}\| \leq \epsilon / 4+\epsilon / 8<\epsilon .
$$

This completes the proof of the theorem.

Proof of Theorem 3.3. By Proposition 5.1 there is $M_{1}>0$ such that for each program $\{x(t), y(t)\}_{t=0}^{\infty}$ satisfying $x(0) \leq M_{0} e$ the following inequality holds:

$$
x(t) \leq M_{1} e \text { for all integers } t \geq 0 .
$$

Theorem 3.2 guarantees that there exists $\delta>0$ such that for each optimal program $\{x(t), y(t)\}_{t=0}^{\infty}$ satisfying $\|x(0)-\widehat{x}\| \leq \delta$ the following inequality holds:

$$
\|x(t)-\widehat{x}\|,\|y(t)-\widehat{x}\| \leq \epsilon \text { for all integers } t \geq 0 .
$$


We show that there exists a natural number $\tau_{0}$ such that the following property holds:

(P) For each optimal program $\{x(t), y(t)\}_{t=0}^{\infty}$ satisfying $x(0) \leq M_{0} e$ there exists an integer $t$ such that

$$
0 \leq t \leq \tau_{0} \text { and }\|x(t)-\widehat{x}\|<\delta
$$

Let us assume the converse. Then for each natural number $k$ there exists an optimal program $\left\{x^{(k)}(t), y^{(k)}(t)\right\}_{t=0}^{\infty}$ such that

$$
x^{(k)}(0) \leq M_{0} e,\left\|x^{(k)}(t)-\widehat{x}\right\| \geq \delta, t=0, \ldots, k .
$$

Proposition 5.4 implies that there is $D_{0}>0$ such that

$$
\Delta(z) \leq D_{0} \text { for each } z \in R_{+}^{n} \text { satisfying } z \leq M_{0} e .
$$

In view of (6.39), (6.38) and Theorems 3.1, 2.2 and 2.3 for each natural number $k$

$$
\sum_{t=0}^{\infty} \delta\left(x^{(k)}(t), y^{(k)}(t), x^{(k)}(t+1)\right)=\Delta\left(x^{(k)}(0)\right) \leq D_{0}
$$

By the choice of $M_{1}$ and (6.38)

$$
x^{(k)}(t), y^{(k)}(t) \leq M_{1} e \text { for all integers } t \geq 0 \text { and all natural numbers } k .
$$

Extracting subsequences, reindexing and using diagonalization process we obtain that there exists a strictly increasing sequence of natural numbers $\left\{k_{j}\right\}_{j=1}^{\infty}$ such that for each integer $s \geq 0$ there exists

$$
(\tilde{x}(s), \tilde{y}(s))=\lim _{j \rightarrow \infty}\left(x^{\left(k_{j}\right)}(s), y^{\left(k_{j}\right.}(s)\right) .
$$

It is not difficult to see that $\{\tilde{x}(t), \tilde{y}(t)\}_{t=0}^{\infty}$ is a program. In view of (6.41), (6.40) and the continuity of the function $\delta(\cdot, \cdot, \cdot)$

$$
\sum_{t=0}^{\infty} \delta(\tilde{x}(t), \tilde{y}(t), \tilde{x}(t+1)) \leq D_{0} .
$$

This implies that $\{\tilde{x}(t), \tilde{y}(t)\}_{t=0}^{\infty}$ is a good program. Theorems 2.2 and 2.3 imply that

$$
\lim _{t \rightarrow \infty} \tilde{x}(t)=\widehat{x} .
$$

On the other hand it follows from (6.38) and (6.41) that

$$
\|\tilde{x}(t)-\widehat{x}\| \geq \delta \text { for all integers } t \geq 0 .
$$

The contradiction we have reached proves that there is a natural number $\tau_{0}$ such that property $(\mathrm{P})$ holds. 
Now assume that $\{x(t), y(t)\}_{t=0}^{\infty}$ is an optimal program satisfying $x(0) \leq M_{0} e$. By property $(\mathrm{P})$ there is an integer $t_{0} \in\left[0, \tau_{0}\right]$ such that

$$
\left\|x\left(t_{0}\right)-\widehat{x}\right\|<\delta
$$

Clearly the program $\left\{x\left(t+t_{0}\right), y\left(t+t_{0}\right)\right\}_{t=0}^{\infty}$ is also optimal. In view of (6.42) and the choice of $\delta$ (see (6.37))

$$
\left\|x\left(t+t_{0}\right)-\widehat{x}\right\|,\left\|y\left(t+t_{0}\right)-\widehat{x}\right\| \leq \epsilon \text { for all integers } t \geq 0 .
$$

This completes the proof of the theorem.

Acknowledgments. Khan acknowledges useful correspondence with Lionel McKenzie, Kazuo Nishimura and Makoto Yano concerning McKenzie (1999); and also the fact, evident from the references in the text, that his thinking of the RSS model has been formed and shaped by his collaboration with Tapan Mitra on the RSS model that began in llinois in 1986. The authors are also especially indebted to one of the anonymous referees for pushing us to emphasize the broader methodological relevance of the results reported here.

\section{REFERENCES}

P. Aghion and S. N. Durlauf (eds.), Handbook of Economic Growth, Volumes $1 A$ and 1B, North Holland, Amsterdam, 2005.

H. Atsumi, Neoclassical growth and the efficient program of capital accumulation, Review of Economic Studies 32 (1965), 127-136.

D. Cass, Optimum growth in an aggegative model of capital accumulation:, Review of Economic Studies 32 (1965), 233-240.

D. Cass, Optimum growth in an aggegative model of capital accumulation: a turnpike theorem, Econometrica 37 (1966), 833-850.

R. Dorfman, P. A. Samuelson and R. M. Solow, Linear Programming and Economic Analysis, McGraw Hill, New York, 1958.

J. Benhabib and B. Hobijn, Another view of investment: forty years later, Knowledge, Information and Expectations in Modern Macroeconomics: in Honor of Edmund S. Phelps (P. Aghion, R. Frydman, J. Stiglitz and M. Woodford eds.), Chapter 26, pp. 522-545 (2003), Princeton University Press, Princeton.

J. Benhabib and A. Rustichini, Vintage capital, investment and growth, Journal of Economic Theory 55 (1991), 323-339.

J. Benhabib and A. Rustichini, A vintage model of growth and investment: theory and evidence, General Equilibrium, Growth and Trade II (R. Becker, M. Boldrin, R. Jones and W. Thomson eds.), pp. 248-3?? (1993), Academic Press, San Diego.

J. Benhabib and A. Rustichini, A note on a new class of solutions to dynamic programming problems arising in economic growth 18 (1994), 808-813.

J. Benhabib and A. Rustichini, A note on a new class of solutions to dynamic programming problems arising in economic growth 18 (1994), 808-813.

W.A. Brock, On existence of weakly maximal programmes in a multi-sector economy, Review of Economic Studies 37 (1970), 275-280.

V. V. Chari and H. Hopenhyn, Vintage human capital, growth and the diffusion of new technology, Journal of Political Economy 99 (1991), 1142-1165.

A. Dixit, Growth patterns in a dual economy, Oxford Economic Papers 22 (1970), 229-234.

D. Gale, On optimal development in a multi-sector economy, Review of Economic Studies 34 (1967), 1-18.

S. Gilchrist and J. Williams, Putty-clay and investment: a business cycle analysis, Journal of Political Economy 108 (2000), 928-60.

B. Jovanovic, Vintage capital and inequality, Review of Economic Dynamics 1 (1998), 497-530. 
M. Ali Khan, Srinivasan on choice of technique again, Trade, Growth and Development: Essays in Honor of Professor T.N. Srinivasan (G. Ranis and L.K. Raut eds.), Chapter 2 (2000), North Holland, Amsterdam.

M. Ali Khan and T. Mitra, Optimal Growth under Irreversible Investment: The Strictly Concave Utility Case, Johns Hopkins University, mimeo (September 17, 2002).

M. Ali Khan and T. Mitra, Optimal Growth in the Two-Sector RSS Model: A Continuous Time Analysis, Johns Hopkins University, mimeo. (Also in Proceedings of the Seventh Portugese Conference on Automatic Control, Lisboa, September 11-13, 2006) (July, 2003).

M. Ali Khan and T. Mitra, On choice of technique in the Robinson-Solow-Srinivasan model, International Journal of Economic Theory 1 (2005a), 83-109.

M. Ali Khan and T. Mitra, Optimal Growth in a Two-Sector Model without Discounting: A Geometric Investigation, Japanese Economic Review, accepted (2005b).

M. Ali Khan and T. Mitra, Undiscounted optimal growth under irreversible investment: a synthesis of the value-loss approach and dynamic programming, Economic Theory, accepted (2005c).

T. C. Koopmans, On the concept of optimal economic growth, The Econometric Approach to Development Planning (Pontificae Academiae Scientiarum Scripta Varia No. 28, pp. 225-287 (1965), North Holland, Amsterdam.

L.W. McKenzie, Accumulation programs of maximum utulity and the von Neumann facet, Value, Capital and Growth (J.N. Wolfe, ed.) (1968), Edinburgh University Press, Edinburgh, 353383.

L.W. McKenzie, Turnpike theory, Econometrica 43 (1976), 841-865.

L.W. McKenzie, Optimal economic growth, turnpike theorems and comparative dynamics, Handbook of Mathematical Economics (K. J. Arrow and M. Intrilligator, eds.) 3 (1986), NorthHolland Publishing Company, New York, 1281-1355.

L.W. McKenzie, Turnpike theory, The New Palgrave (J. Eatwell, M. Milgate and P. K. Newman, eds.) 4 (1987), MacMillan Publishing Company, New York, 712-720.

L.W. McKenzie, Turnpikes, American Ecoomic Review (Papers and Proceedings) 88 (1998), 1-14.

L.W. McKenzie, Equilibrium, trade and capital accumulation, The Japanese Economic Review 50 (1999), 369-397.

L.W. McKenzie, Classical General Equilibrium Theory, The MIT Press, Cambridge, 2002.

T. Mitra, On optimal economic growth with variable discount rates: existence and stability results, International Economic Review 20 (1979), 133-145.

T. Mitra, Characterization of the turnpike property of optimal paths in the aggregative model of intertemporal allocation, International Journal of Economic Theory 1 (2005), 247-275.

T. Mitra, K. Nishimura and G. Sorger, Optimal cycles and chaos, Handbook of Growth (C. le Van (ed.)), forthcoming.

T. Mitra and I. Zilcha, On optimal economic growth with changing technology and tastes: characterization and stability results, International Economic Review 22 (1981), 221-238.

R. Radner, Paths of economic growth that are optimal only with respect to final states, Review of Economic Studies 28 (1961), 98-104.

F. Ramsey, A mathematical theory of savings, Economic Journal 38 (1928), 543-559.

J. Robinson, Exercises in Economic Analysis, MacMillan, London, 1960.

P. A. Samuelson, A catenary turnpike theorem involving consumption and the golden rule, American Economic Review 55 (1965), 486-496.

P. A. Samuelson, Optimality of profit, including prices under ideal planning, Proceedings of the National Academy of Sciences USA 70 (1973), 2109-2111.

P. A. Samuelson and R. M. Solow, A complete capital model involving heterogeneous capital goods, Quarterly Journal of Economics LXX (1956).

R.M. Solow, Investment and technical progress, Mathematical Methods in the Social Sciences (K. J. Arrow, S. Karlin and P. Suppes eds.), Chapter 1 (1960), Stanford University Press, Palo Alto.

R.M. Solow, Substitution and fixed proportions in the theory of capital, Review of Economic Studies 29 (1962), 207-218.

R.M. Solow, Srinivasan on choice of technique, Trade, Growth and Development: Essays in Honor of Professor T.N. Srinivasan (G. Ranis and L.K. Raut eds.), Chapter 1 (2000), North Holland, Amsterdam.

R.M. Solow, Reflections on growth theory, Handbook of Economic Growth (P. Aghion and S. N. Durlauf eds.), pages 3-10 (2005), North Holland, Amsterdam. 
T.N. Srinivasan, Investment criteria and choice of techniques of production, Yale Economic Essays 1 (1962), 58-115.

J.E. Stiglitz, A note on technical choice under full employment in a socialist Economy, Economic Journal 78 (1968), 603-609.

J.E. Stiglitz, Recurrence of techniques in a dynamic economy, Models of Economic Growth (J. Mirrlees and N. H. Stern eds.) (1973), John-Wiley and Sons, New York, 138-161.

von J. Neumann, A model of general economic equilibrium, Review of Economic Studies 13, 1-9.

C.C. von Weizsacker, Existence of optimal programs of accumulation for an infinite horizon, Rev. Econ. Studies 32 (1965), 85-104.

A.J. Zaslavski, Optimal programs in the RRS model, International Journal of Economic Theory 1 (2005), 151-165.

Department of Economics, The Johns Hopkins University, Baltimore, MD 21218 , USA

Department of Mathematics, The Technion-Israel Institute of Technology, 32000 HAIFA, ISRAEL

E-mail address: akhan@jhu.edu; ajzasl@tx.technion.ac.il 